\title{
Quantification of energy savings from dynamic solar radiation regulation strategies in office buildings
}

\author{
Alex Vlachokostas ${ }^{1}$, Nicholas Madamopoulos ${ }^{1,2}$ \\ ${ }^{1}$ Department of Electrical Engineering \\ The City College of City University of New York \\ New York, NY 10031, United States \\ ${ }^{2}$ Hellenic Air Force Academy, Department of Aeronautical Sciences \\ Dekelia, 13761 (1010), Greece
}

\begin{abstract}
:
Traditionally, glazing-specific technologies have been studied at a theoretical or experimental level with the goal of identifying the optimum thermo-optical parameters that the glazing should have in order to provide energy savings. In this work, we focus on quantifying the impact of the direct and diffuse solar radiation components on energy savings for office buildings following a technology-agnostic approach. More specifically, the question to be answered is: "How can independent management of the solar radiation components offset electric lighting, but on the other hand, possibly put additional load requirements on space-heating/cooling, and fans?" We use EnergyPlus to simulate an adiabatic perimeter office space, with typical zone characteristics, under various solar radiation levels. The solar radiation values are derived from an EnergyPlus weather file. We process that original weather file and create new weather files with reduced solar radiation by specific percentages and simulate the same adiabatic office. We only reduce the direct and diffuse solar radiation components of the original weather file in order to study the impact of regulated solar radiation on the zone's lighting, heating, fans, and cooling primary energy requirement. Second, we perform a dynamic solar radiation regulation analysis on a monthly, daily, and hourly basis. We show that faster regulation response time of the solar radiation leads to higher energy savings. The reference scenarios for the North, East, South, and West-facing zones require a total of $71,106,87$, and $99 \mathrm{kWh} / \mathrm{m}^{2} \mathrm{y}$ respectively. An hourly solar radiation regulation can lead to maximum energy savings of $18 \%, 33 \%, 37 \%$ and $36 \%$ for each orientation. Finally, we perform a study that quantifies the impact of potential technological constraints, such as reduced dynamic range and resolution of the solar radiation admittance, on energy savings.
\end{abstract}

Keywords: dynamic regulation strategies; energy savings; solar radiation impact; direct and diffuse solar radiation components.

\section{Introduction}

According to Negawatt Revolution by Amory Lovins "The best energy policy for the nation, for business, and for the environment is one that focuses on using electricity efficiently" [1]. The top energy-intensive building end-uses, which are electric lighting, space heating and cooling, and fans are mainly responsible for the total primary energy requirement (TPER) [2, 3]. It is well known that these building end-use functions are highly affected by solar radiation. Solar radiation availability and intensity is a major energy consumption driver, especially in glazingdominated commercial buildings. More specifically, excess solar radiation leads to more cooling demand in the summer time, while scarcity of solar radiation leads to higher heating demand in 
the winter time. In addition, solar radiation availability affects the balance between electric lighting and natural daylight. Therefore, appropriate daylight offsets the need for electric lighting, whereas the absence of daylight drives the electric lighting energy consumption. Quantifying the physical interplay between solar radiation and building end-uses is very important, since admitting and blocking solar radiation can have adverse effects on the zone's energy requirements.

A considerable number of studies have evaluated the effect of solar radiation on the zone's energy consumption. Favoino et al. [4] investigated glazing systems that change their thermooptical properties, on a monthly and daily basis, in order to reduce the energy use in buildings. Hammad et al. [5] explored how dynamic external louvers can lead to energy savings by regulating admitted solar radiation. Susorova et al. [6] studied how geometry factors, such as window sizing and room depth affect the total energy consumption in an office. Similarly, Tzempelikos et al. [7] parameterized the window-to-wall ratio and studied the effect on total energy consumption, daylight, and peak energy use. Ihara et al. [8] explored how thermal quantities, such as façade U-Value and solar heat gain coefficient (SHGC), affect energy savings in office buildings. Lastly, Chua et al. [9] studied the relation between energy consumption and the envelope thermal transfer value. All these studies presented how technology-specific elements affect incoming solar radiation and hence play an important role in the office energy consumption. On the contrary, in our approach we follow a technology-agnostic methodology. In other words, we don't simulate specific thermo-optical glazing properties, particular lightredirecting devices, window and office room geometry, or any envelope insulation properties. We simulate the same adiabatic office space under various admitted solar radiation conditions. We do that in order to understand how solar radiation affects the zone's primary energy requirement and suggest solutions to reduce the zone's energy needs. This approach allows us to better understand the physical interplay between admitted solar radiation and energy needs and develop recommendations and/or derive specifications for next generation dynamic fenestrations without focusing on the technology but rather on the performance requirements. The focus of our work is to quantify the impact of daylighting (e.g., admittance levels of diffuse and direct solar radiation) in the energy requirements without the impact of any additional glazing properties. Hence, we do not vary any thermo-optical glazing properties.

In this paper, we show that the independent regulation of the two solar radiation components (direct and diffuse) affects differently the energy consumption and if appropriately managed/regulated provides additional energy saving benefits to offices. The outcome of the study explores:

(i) The response time of dynamic regulation of the incoming solar radiation,

(ii) The optimum dynamic range of solar radiation regulation, and

(iii) The energy saving impact of solar radiation dynamic range and resolution limitations.

To our knowledge, this is the first time that such technology-agnostic quantification has been performed.

The paper is organized as follows: In Section 2, we present the methodology and assumptions of our study. In Section 3, we present the simulation results starting from a few simulated scenarios (i.e., 55) in order to show patterns of how the direct and diffuse solar radiation affect the zone's energy requirement. In Section 4 we expand our study to the total number of simulated scenarios 
(i.e., 16,744). More specifically, in Section 4.1 we show the importance a dynamic regulation strategy (DRS) of solar radiation and apply a monthly, daily, and hourly DRS in order to achieve energy savings. In Section 4.2 we discuss the impact of solar radiation resolution and dynamic range (DR) admittance levels on energy savings. Finally, Section 5 summarizes the findings in a conclusion.

\section{Methodology and assumptions}

Our methodology is based on simulating an adiabatic office space under different solar radiation admittance scenarios [10]. In particular, instead of modifying the thermo-optical fenestration parameters we treat the fenestration as a "black box". This "black box" approach is based on the modification of the original weather file (e.g., .epw file) downloaded from the EnergyPlus Weather Data-U.S. Department of Energy [11]. We process the original weather file and create new weather files for which we reduced direct and diffuse solar radiation values by specific percentages. Note that since we have access to both direct and diffuse solar radiation at the weather file level, we can independently change them and thus simulate different scenarios by reducing the direct and/or diffuse solar radiation values at the weather file level. By doing so, we are emulating a potential reduction/regulation of solar radiation from the glazing that produces the desired level of admitted solar radiation to the zone. Fig. 1(a) shows a typical perimeter office space, where the incoming direct and diffuse solar radiation is regulated by a daylight control device. Fig. 1(b) shows a schematic of our methodology, in which the fenestration and any additional daylighting device is lumped as a "black box". This "black box" regulates the incoming solar radiation and achieves the same incoming direct and diffuse solar radiation as in the case of Fig. 1(a). In this paper, we do not consider what technology (e.g., electrochromic glazing, etc) can emulate such an effect. Our goal is to understand and quantify the basic interaction between regulated solar radiation and the zone's energy consumption. For that reason, we change only the solar radiation, while keeping the same glazing system, office materials, etc.

Our reference scenario is the adiabatic perimeter office that is simulated with the original weather file. Then we simulate a series of scenarios, where we change the solar radiation at the weather file level in steps of $1 \%$ and keep the same adiabatic office space. In our methodology, we selectively reduce the two components of the incoming solar radiation. In the reference scenario, we use the original, unmodified weather file (e.g., reference.epw) and the EnergyPlus file (e.g., .idf file), which describes all building and occupant related data. In all other scenarios, the weather file is modified (e.g., scenario.epw) and in conjunction with the EnergyPlus file the energy performance of the adiabatic office is simulated over and over again $(4,186$ times per cardinal orientation) for all different weather file inputs.

To limit computational time and to eliminate multiple contributions of other energy-related sources in a larger building setting, we model only one perimeter office space as a single thermal zone, and not an entire building. Care must be taken when modeling a single thermal zone. All walls, except from the front façade, must be treated as adiabatic in order to eliminate thermal contribution from the environment [12]. An adiabatic wall is defined as one that does not permit heat transfer between the outdoor and indoor environment. However, this can only be implemented for all the surrounding walls in the zone (e.g., floor, ceiling, side and back wall) but not for the façade wall. This is because the façade wall is a "host-surface" for the glazing and 
"host-surfaces" in EnergyPlus cannot be adiabatic. As a result, we must define the facade wall

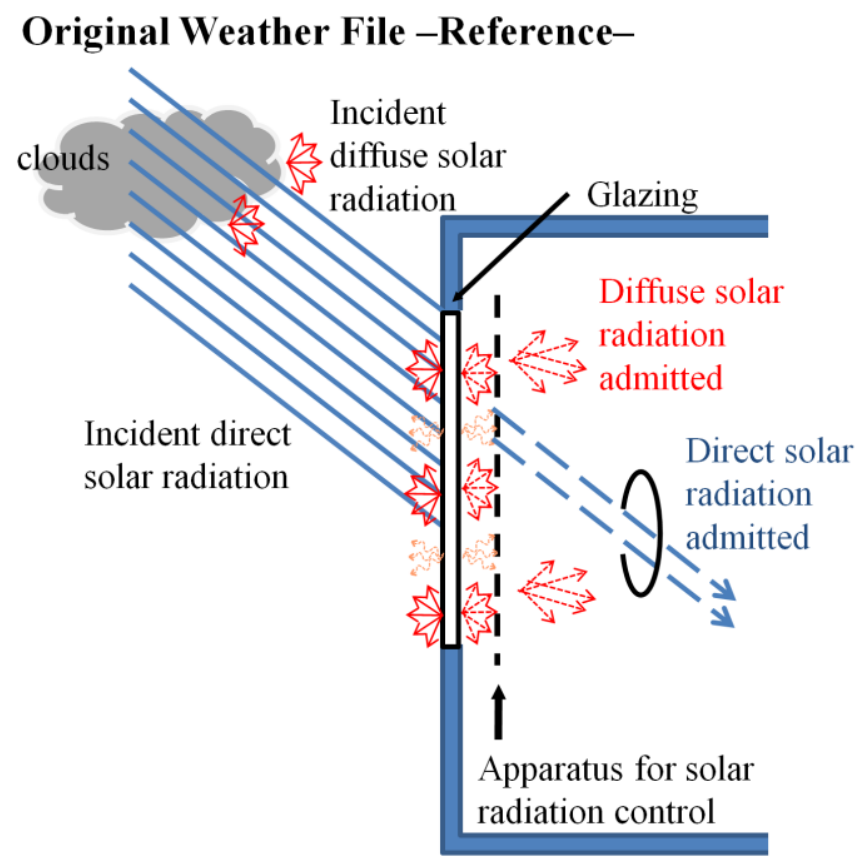

(a)

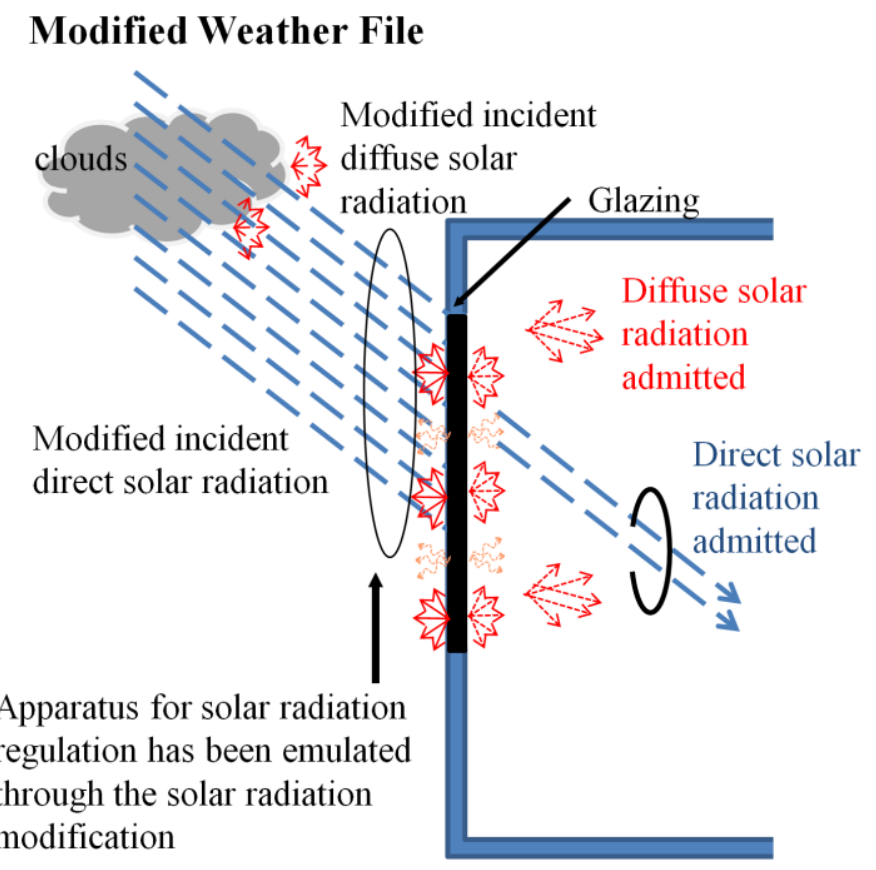

(b)

Fig. 1. (a) Reference simulation. The adiabatic office is simulated with the original weather file, (b) modified solar radiation entering the same adiabatic office. In both cases the admitted diffuse and direct solar radiation levels are equivalent. 
with a very high $R$-value. Since in our methodology, we modify the solar radiation that enters the office space, a "host-surface" wall with typical $R$-value may contribute in heat gains for the modified weather files. Therefore, in order to minimize heat transfer through the façade wall and account only for the effect of the admitted solar radiation through the glazing, we have intentionally used an artificially high $R$-value (e.g., $>1000 \mathrm{~K} \cdot \mathrm{m}^{2} / \mathrm{W}$ ) so that heat transfer through the façade wall does not contribute to the associated heating and/or cooling requirements of the thermal zone. We evaluated the contribution of the heat gain from the fenestration and the façade wall, respectively, and we found that the heat gain from the façade wall is only $0.7 \%$ of the heat gain from the glazing for the East orientation. The other orientations have even smaller contributions. As a result, the heat gain contribution from the façade wall is minimal when compared to the heat gain from the glazing. Therefore, those percentages can be considered as a safe error margin $(<1 \%)$ in our attempt to quantify only the heat gain contribution from the glazing. Last, in an actual building, the building materials have heat capacity. This may delay a response to changes in the settings of the fenestration system. However, our modeling work makes two assumptions: (a) the walls do not transfer heat, and (b) the walls have no heat capacity, so that the temperature of the space changes entirely in response to exterior forcings (i.e., solar radiation). These assumptions are made for evaluating only the impact of admitted solar radiation components to the energy consumption of the zone.

In our study, we created various simulation scenario groups. The first simulation group keeps the diffuse solar radiation at $100 \%$ (meaning that the diffuse solar radiation value is the same as that of the original weather file), whereas the direct solar radiation varies from $100 \%$ to $10 \%$ at steps of $1 \%$. The second simulation group uses a reduced diffuse solar radiation value, which is $99 \%$ of the diffuse solar radiation value of the original weather file. Direct solar radiation for the second group varies from $99 \%$ to $10 \%$ in steps of $1 \%$. Additional scenarios are obtained by further reducing the diffuse solar radiation and accordingly reducing the direct solar radiation as well. As a result, a total of 4,186 scenarios are obtained for each zone orientation. The reference scenario, i.e., $S_{100,100}$, is obtained by simulating the adiabatic office space with the original weather file. All the scenarios used in our study, which are labeled as $S_{\mathrm{i}, \mathrm{j}}$, where $i$ is the index showing the direct solar radiation admittance level and $j$ is the index showing the diffuse solar radiation admittance level. For example, the $S_{10,98}$ scenario means that the adiabatic office space was simulated with a weather file whose direct solar radiation component is $10 \%$ of the value of the original and its diffuse solar radiation component is $98 \%$ of the values of original weather file, hence, it emulates a daylight device that regulates the incoming solar radiation to admit $10 \%$ and $98 \%$ of the incoming direct and diffuse solar radiation. For the scope of this paper, we assume that a fenestration technology admits/reduces the diffuse solar radiation to a value of $X \%$ of the available one from the outdoors environment and that the direct solar radiation cannot exceed this $X \%$. However, in future work we plan to include cases where direct solar radiation is higher than diffuse. We limit the minimum admittance level of direct and diffuse solar radiation to $10 \%$ because EnergyPlus malfunctions and develops errors for simulation files that contain solar radiation at values lower than $10 \%$ of the original file.

Our study does not consider whether or not such selective control of the solar radiation can be accomplished with today's technology. Our focus is to:

1. Study and understand the impact of such an effect; 
2. Derive optimum conditions. That means which solar radiation condition enhances energy savings; and

3. Derive performance specifications targets for next-generation dynamic glazings independent of the technology.

The proposed perimeter office space is a standard $3 \mathrm{~m} \times 6 \mathrm{~m}$ (width $\times$ depth) office, with a floor to ceiling height of $2.75 \mathrm{~m} \mathrm{[13].} \mathrm{The} \mathrm{lighting} \mathrm{system} \mathrm{is} \mathrm{continuously} \mathrm{dimmable} \mathrm{to} \mathrm{compensate}$ for daylighting illuminance. Target illumination is 500 lux at the work plane, which is located $0.8 \mathrm{~m}$ above floor. The light control sensors are located at $2 \mathrm{~m}$ and $4 \mathrm{~m}$ from the window. The electric lighting consists of $72 \%$ long-wave (thermal) radiation, $18 \%$ visible (short-wave) radiation, and $10 \%$ is convected [14]. We used typical interior surface reflectance values for the floor, ceiling, and walls of $20 \%, 50 \%$, and $80 \%$, respectively [15]. Occupant density in the space is 0.111 people $/ \mathrm{m}^{2}$, and sensible heat from each occupant is $70 \mathrm{~W}$ [15]. Heating and cooling are always available in the office space throughout the year and fans follows the heating and cooling schedule [15]. The majority of commercial buildings in the US do not have operable windows, due to safety concerns. Natural outside air is delivered to the building through Air Handling Units (AHUs) and air duct equipment. Therefore, our thermal zone does not consider an operable window setup. Our current approach does not address the cases of corner offices/thermal zones, which have windows facing in different orientations. However, in large commercial buildings, the number of corner offices is much lower than the offices with windows facing only one orientation. Table 1 shows the additional simulation assumptions involving the HVAC system, thermostat set-points, electric loads, and window type.

New York City (NYC) has a mixed-humid climate (ASHRAE Climate Zone 4A, [16]) with a daily average dry-bulb temperature of $1{ }^{\circ} \mathrm{C}$ in January and $24{ }^{\circ} \mathrm{C}$ in July. As a metropolitan city, $\mathrm{NYC}$ has a lot of heat-holding elements such as buildings, streets, and pavements and fewer trees compared to rural areas. Because NYC is significantly warmer than its surrounding rural areas; the NYC climate has urban heat island (UHI) characteristics.

Table 1. Actual end-use load technologies.

\begin{tabular}{|c|c|}
\hline System & Parameters \\
\hline HVAC & $\begin{array}{c}\text { Single zone direct expansion } \\
\text { Cooling: COP } 4.5, \text { Heating: eta } 0.95 \\
\text { Flow rate: } 0.01\left(\mathrm{~m}^{3} / \mathrm{s}\right) / \text { person }\end{array}$ \\
& Fuel: natural gas (heating) and electricity (cooling) \\
\hline Thermostat set-points & $24^{\circ} \mathrm{C}$ for cooling, $21^{\circ} \mathrm{C}$ for heating \\
Night setback: $28^{\circ} \mathrm{C}$ for cooling, \\
$16{ }^{\circ} \mathrm{C}$ for heating
\end{tabular}




\section{Simulation results}

We start by presenting, in Fig. 2, only 55 out of 4,186 scenarios in order to clearly show the energy consumption trends (e.g., reduction or increase) when we vary solar radiation. The simulations in Fig. 2 are organized in ten distinct groups for clarity. First we present the reference scenario (i.e., $S_{100,100)}$ ) by itself. Then we present the first group of simulations, in which the direct solar radiation admitted into the office space varies from $90 \%$ to $10 \%$ of the original weather file value, in steps of $10 \%$, whereas the diffuse solar radiation is set constantly at $100 \%$. The next group presents the results for scenarios with diffuse solar radiation constantly set at $90 \%$ and with direct solar radiation varying from $90 \%$ to $10 \%$ of the original weather file value, in steps of $10 \%$. Accordingly, the other scenario groups are presented in Fig. 2. The $y$ axis represents the annual total primary energy requirement (i.e., TPER) expressed in $\mathrm{kWh} / \mathrm{m}^{2} \mathrm{y}$ and the $x$-axis represents the simulation scenario groups. We plot the primary energy requirement for the lights, heating, fans, and cooling in a stacked-bar fashion to better visualize and understand the impact of the two solar radiation components on the individual building enduse functions and the zone's primary energy needs. Note that TPER calculations use different conversion rates for electricity and natural gas. More specifically, $1 \mathrm{KWh}$ of electricity is counted as $2.18 \mathrm{kWh}$ and $1 \mathrm{kWh}$ of natural gas as $1.1 \mathrm{kWh}$ when calculating the TPER versus the total final consumption (TFC) [17].

Fig. 2(a) shows that the reference North-facing zone scenario consumes the least amount of energy (i.e., $71 \mathrm{kWh} / \mathrm{m}^{2} \mathrm{y}$ ), when compared to the other reference orientations. For this zone, electric lighting requirements are the highest, but air-conditioning requirements are minimal. The reason that no intensive air-conditioning is needed is that the North-facing zone is not exposed to direct solar radiation. The lack of direct solar radiation results in less heat gain for the Northfacing zone and thus less cooling load demand. In a similar way, Fig. 2(c) shows that the Southfacing zone consumes less energy (i.e., $87 \mathrm{kWh} / \mathrm{m}^{2} \mathrm{y}$ ) when compared to the East (i.e., 106 $\mathrm{kWh} / \mathrm{m}^{2} \mathrm{y}$ ) and West (i.e., $99 \mathrm{kWh} / \mathrm{m}^{2} \mathrm{y}$ ) orientations. More specifically, at the South-facing zone the sun follows an overhead path and direct solar radiation enters the office at a steeper angle. Due to the steeper solar angle, the full amount of solar radiation that enters the South-facing zone is limited and hence there is less solar heat gain when compared to the East and West orientations. On the other hand, at the East and West-facing zones the direct sun enters in the zone area at a lower angle and penetrates deeper into the office space and therefore contributes to the heat gain of the zones. At orientations where there is increased solar heat gain (e.g., East and West), we expect an energy consumption increase due to the air-conditioning operation that actively tries to respond to the solar heat gain induced load. As we notice from Fig. 2(b) and (d), when we reduce the incoming solar radiation at the East and West orientations there is an expected increase in energy consumed by electric lights. On the other hand, for the South orientation (Fig. 2(c)), where there is abundant solar radiation, its regulation does not heavily affect the operation of lights. However, for the North-facing zone, we notice that the regulation of the admitted solar radiation affects the electric lighting energy consumption more intensively. This is an expected result, because the North-facing zone is not exposed to as much direct solar radiation as the other orientations. Therefore, reducing the already scarce natural illumination drives the electric lights to operate more intensively in order to reach the target illumination set by the zone's lighting sensors. 


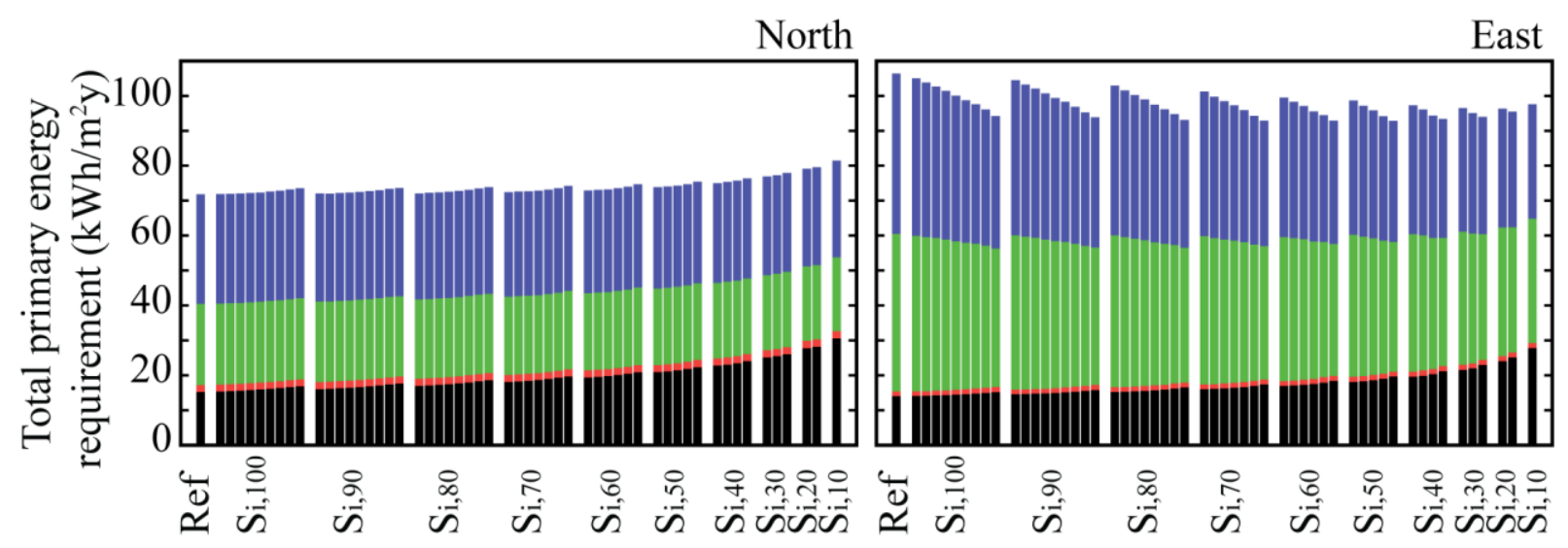

(a)

(b)

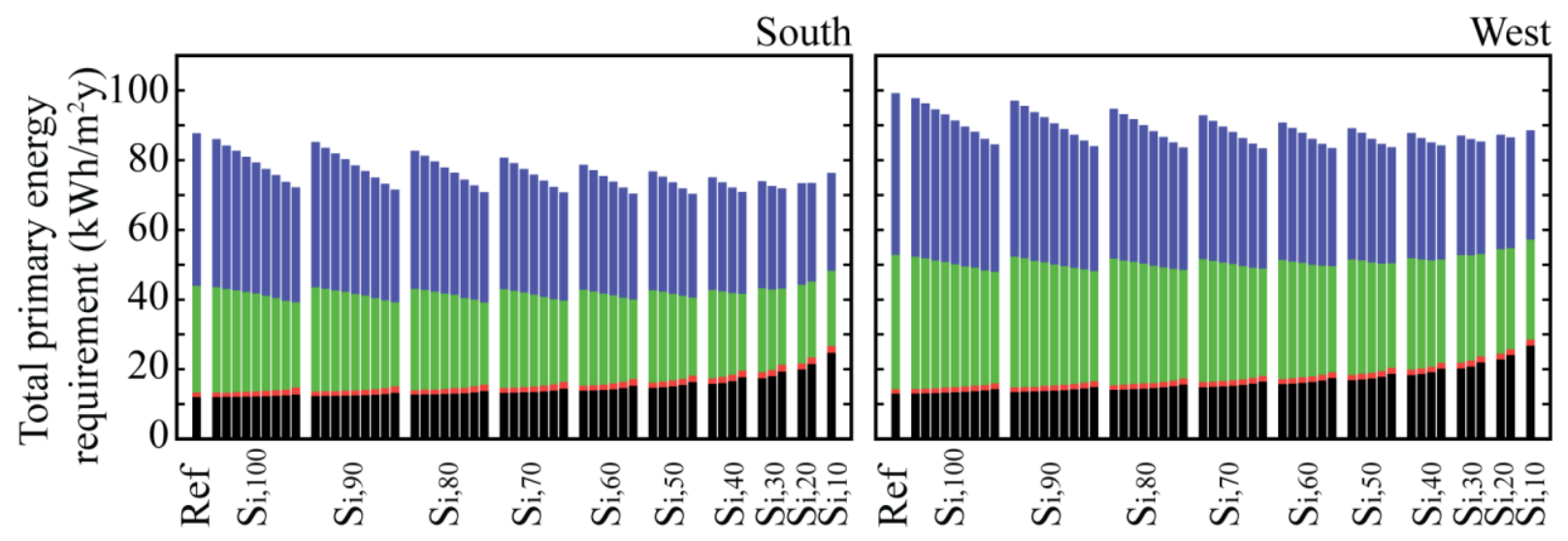

(c)

(d)

- Lights $\square$ Heating $\|$ Fans $\square$ Cooling

Fig. 2. We present the total primary energy requirement in $\mathrm{kWh} / \mathrm{m}^{2} \mathrm{y}$ for (a) North, (b) East (c) South and (d) West-facing perimeter office space under various solar radiation regulation levels. (Note: The index $i$ (direct) varies in steps of $10 \%$ for each scenario group).

Finally, as we notice from the plots in Fig. 2, we can extract the least energy-intensive scenario for each orientation for the 55 presented scenarios. More specifically, for the North orientation the scenario that consumes the least amount of energy is the reference scenario itself. This is essentially the adiabatic office space that is simulated with the original weather file or in other words with a glazing that does not perform any regulation on the incoming solar radiation. On the other hand, for the East, South, and West-facing zones the scenarios with the least energy requirements are $S_{10,50}, S_{10,50}$ and $S_{10,70}$ respectively. Their energy consumption differences from their reference scenarios are $14 \mathrm{kWh} / \mathrm{m}^{2} \mathrm{y}$ (or $13 \%$ reduction), $17 \mathrm{kWh} / \mathrm{m}^{2} \mathrm{y}$ (or $20 \%$ reduction), and $16 \mathrm{kWh} / \mathrm{m}^{2} \mathrm{y}$ (or $16 \%$ reduction), respectively.

\section{Dynamic regulation strategies (DRS) for solar radiation}

\subsection{Dynamic weather conditions and resultant solar radiation regulation}

The results presented in the previous section, represent non-dynamic regulation of the incoming solar radiation. These results showed that with "fixed" or non-dynamic fenestrations, energy 
savings of 10-20\% can be obtained for the East, South, and West-facing zones. However, weather conditions, as well as solar radiation availability, vary throughout the year. Thus, operating the glazing under non-dynamic conditions cannot provide optimum results in energy savings. Fig. 3(a) and Fig. 3(b) show the solar radiation and the temperature profile for a clear sky day (e.g., March $18^{\text {th }}$ ) and an overcast day (e.g., March $17^{\text {th }}$ ) in NYC for a typical meteorological year (TMY3) as given by the original weather file [12]. In Fig. 3(c) and Fig. 3(d) we present the following four scenarios $S_{80,80}, S_{60,60}, S_{40,40}$, and $S_{20,20}$. The $y$-axis shows the total primary energy requirement savings, which is defined as the difference between the zone's total primary energy requirement in the reference scenario and in the simulated scenario (i.e., TPER Ref - TPER $\left.R_{S i, j}\right)$. The results are plotted for each scenario with the solid lines. More specifically, for each hour of the day, we compare the scenario TPER values and select the one that provides the highest total energy savings, while meeting the lighting and heating/cooling set-points. The results are shown in Fig. 3(c) and Fig. 3(d) as open circles. This is identified as the hourly dynamic regulation strategy (DRS[h]) of solar radiation. In principle, this DRS[h] chooses, from among the different scenarios under study or the reference scenario (in the case when no savings are obtained by any of the solar radiation regulated scenarios), the one that requires the least amount of energy. As a result, positive values in Fig. 3(c) and Fig. 3(d) indicate that the respective simulated scenario is saving energy. On the other hand, negative values indicate that the respective scenario is consuming more energy compared to the reference scenario. We can see from Fig 3(c) that during a clear day the energy savings are higher as we admit lower levels of solar radiation into the room. The best energy-performing scenario is consistently $S_{20,20}$. In contrast, during a day that has both overcast and clear periods (e.g., Fig. 3(b)) the best energyperforming scenario is not always the one that admits the least solar radiation. More specifically, we notice the following for the case of March $17^{\text {th: }}$

1. During the morning hours (before $12 \mathrm{pm}$ ), when direct solar radiation is minimal due to clouds (Fig. 3(b)); no reduction in admitted solar radiation provides the optimum results in terms of total primary energy requirement savings for the lights, heating, fans, and cooling. In other words, the $S_{R e f}$ is the best energy performing scenario which shows that no reduction (regulation) of the solar radiation admittance is required.

2. For the latter half of the day (i.e., after 12 pm, Fig. 3(d)), when the direct solar radiation increases significantly, the best energy-performing scenario is the $S_{20,20}$ scenario.

Hence, Fig. 3 shows the importance of a dynamic glazing, which dynamically regulates the admitted solar radiation based on weather conditions. Therefore, in order to achieve maximum energy savings we must consider dynamic solar radiation regulation strategies. To address this challenge and understand the requirements set for the new fenestration technologies, we have developed a methodology that evaluates the energy performance of each $\mathrm{S}_{i, j}$ scenario, on a monthly, daily, and hourly basis. 


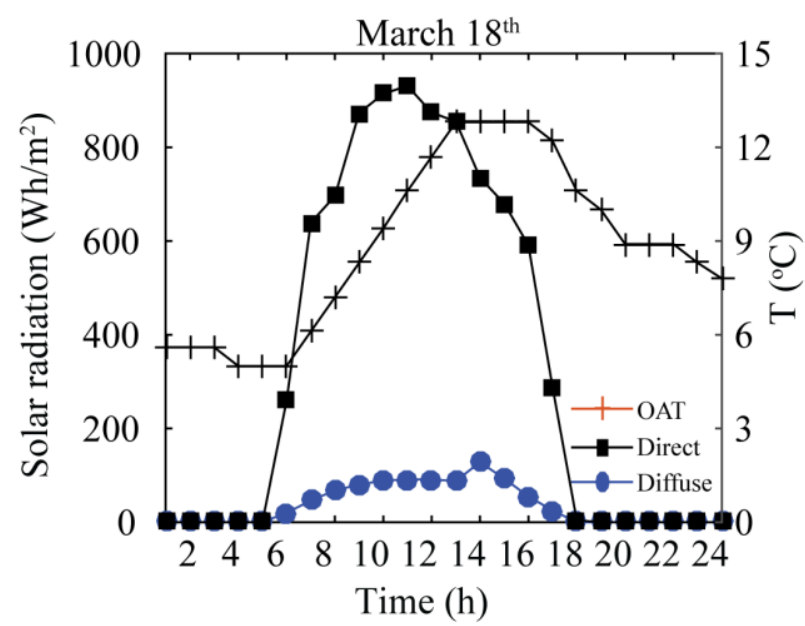

(a)

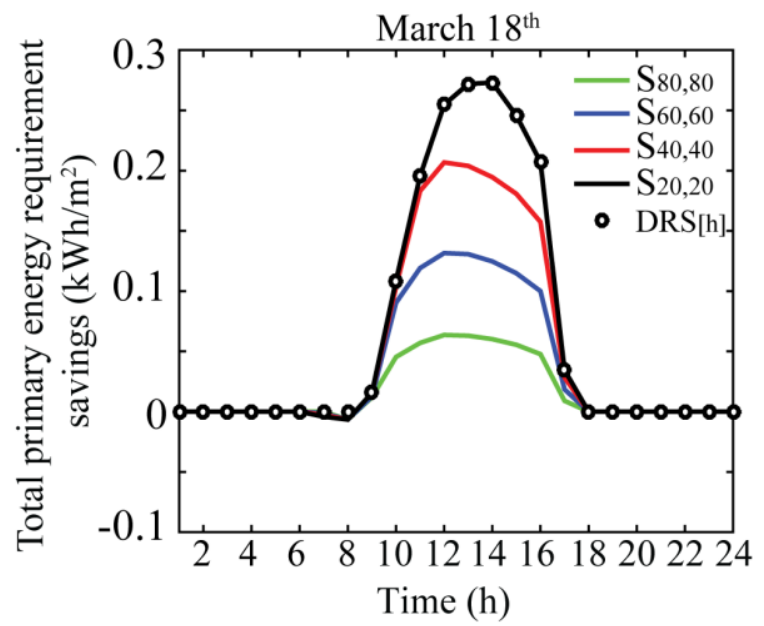

(c)

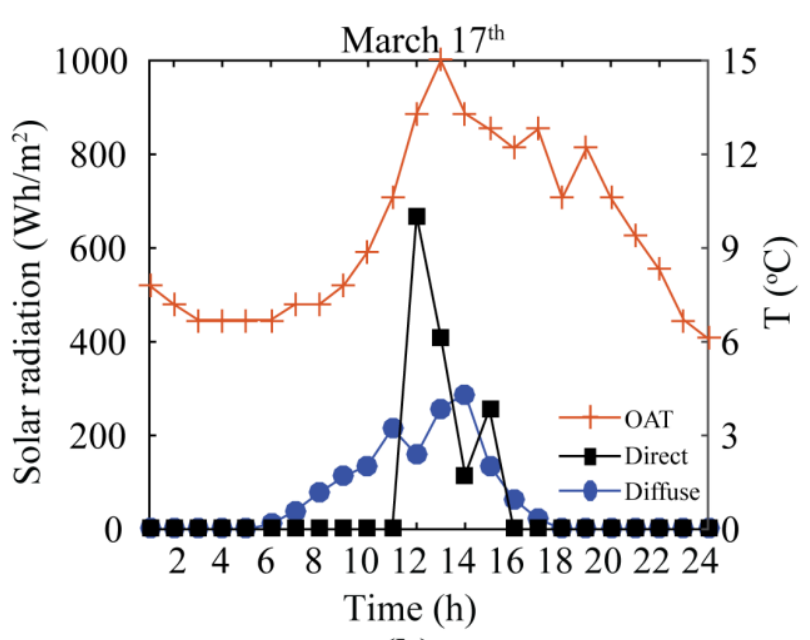

(b)

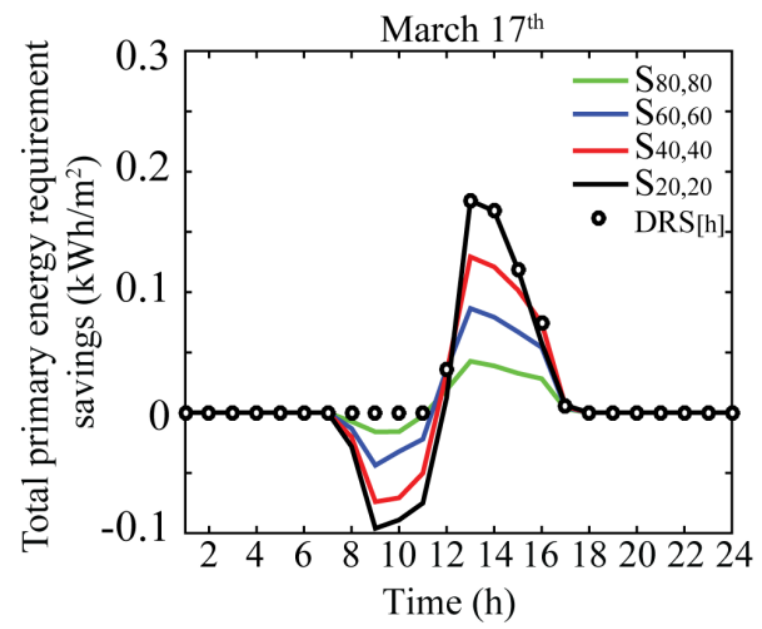

(d)

Fig. 3. TPER for the South-facing zone: Weather conditions for (a) March $18^{\text {th }}$; a clear sky day, and (b) March $17^{\text {th }}$; an overcast day. Four different solar radiation admittance scenarios for (c) March $18^{\text {th }}$, where the best energy-performing strategy is to significantly reduce the admitted solar radiation, and (d) March $17^{\text {th }}$, a combination of solar radiation regulation scenarios offers better results.

The methodology is described in Fig. 4. More specifically, Fig 4(a) shows that each user modified weather file (e.g., .epw file) is simulated with its respective EnergyPlus file (e.g., .idf file). As a result, we have created 4,186 different weather files and all of them have the direct and diffuse solar radiation components reduced. We reduce the amount of admitted solar radiation for the two components at a step of $1 \%$ from the original weather file values [10]. Note that all other EnergyPlus file parameters (i.e., geometry, HVAC system, glazing system, etc) remain the same. Each EnergyPlus simulation output (e.g., $S_{\mathrm{ij}}$ ) is used to perform the postprocessing of the results. Fig. 4(b) shows the methodology pertinent to the monthly dynamic regulation strategy. In particular, first, our methodology calculates the total primary energy requirement (TPER) for each month of each scenario (e.g., Monthly TPER calculation stage). Then it compares the TPER of the same month for all scenarios, and selects the best energyperforming scenario on a per month basis. The best energy-performing scenario corresponds to the solar radiation admittance scenario that provides the maximum energy savings for the office 
space. Finally the overall yearly TPER is calculated using the associated optimum scenarios for each month. We performed a similar set of simulations and post-processing under three different dynamic solar radiation regulation strategies:

1. The first dynamic solar radiation regulation strategy is monthly, DRS[m]. As mentioned above, we evaluate the cumulative energy requirements for the entire month for all scenarios under study. Then, we select as the optimum solar radiation admittance scenario for the entire month, the scenario for which the thermal zone has the maximum energy savings.

2. The second dynamic solar radiation regulation strategy is daily, DRS[d]. The selection of the optimum admittance scenario is based on cumulative results on a daily basis.

3. The third dynamic solar radiation regulation strategy is hourly, DRS[h]. The selection of the optimum admittance scenario is based on cumulative results, where the algorithm selects the best performing scenario for each hour of the day.

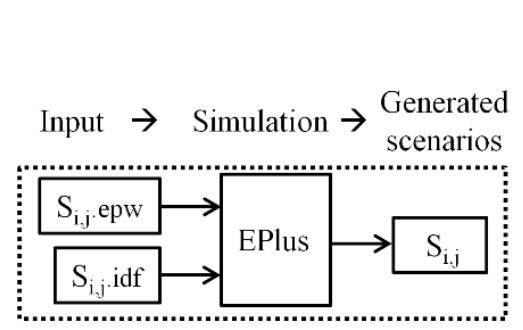

(a)

Post-simulation dynamic regulation strategy (DRS)

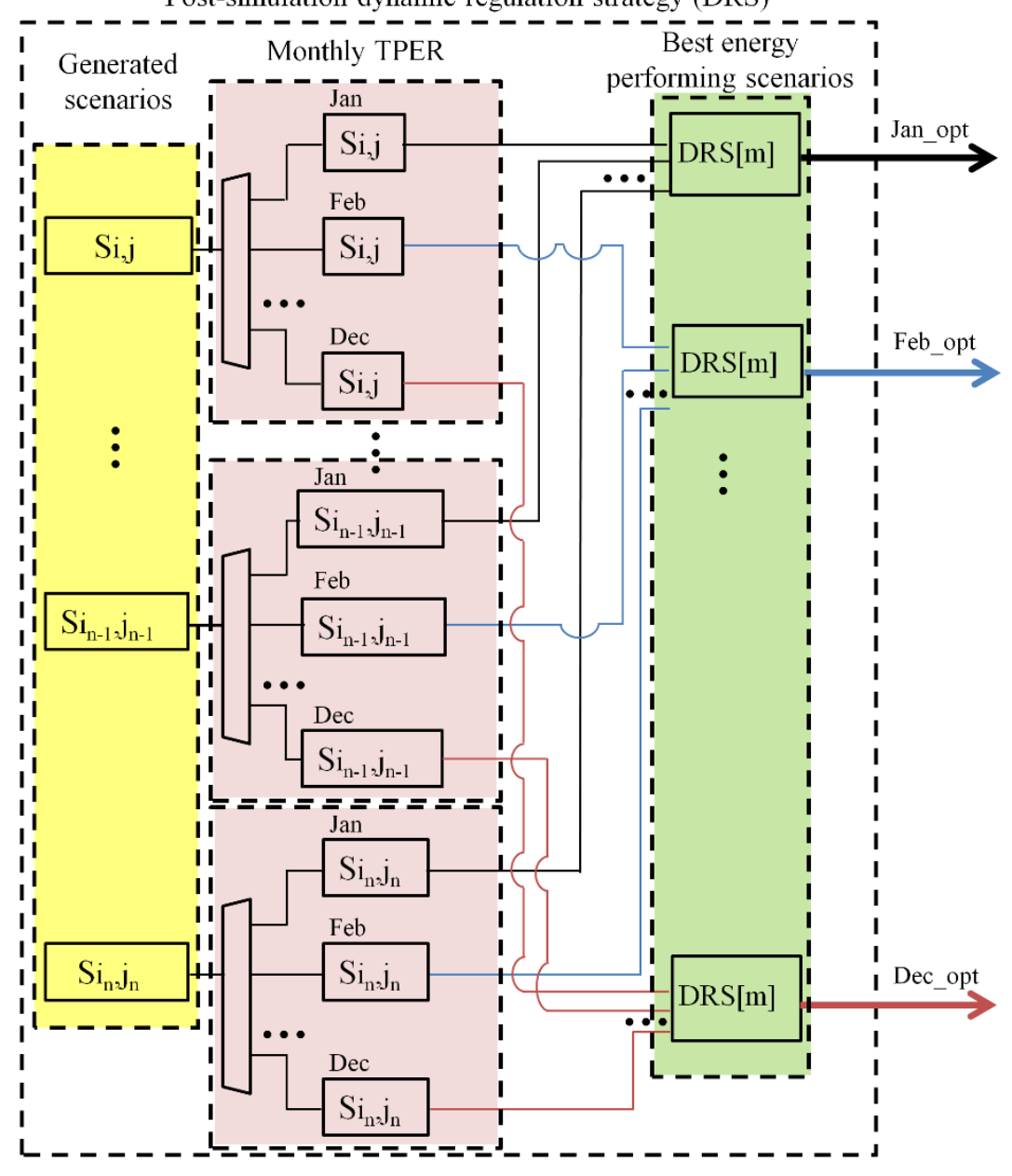

(b)

Fig. 4. (a) Input parameters and (b) monthly dynamic solar radiation regulation strategy DRS[m]. (Note: DRS[d] and DRS[h] follow the same logic). 
The results of this dynamic regulation strategy are presented in Fig. 5, where we have included a comparison among the reference scenario and the dynamic solar radiation regulation strategies of DRS[m], DRS[d], and DRS[h]. These different time bases correspond to different glazing dynamic response times. For example, a DRS[m] may correspond to an installed apparatus/device on a glazing that performs a monthly-set solar radiation reduction for all the days of the month. Such an approach would require changes of the apparatus/device on a monthly basis and the selection should be based on historical data or long term weather predictions. The DRS[d] may correspond to an apparatus/device that performs a daily-set solar radiation reduction. Such a solar radiation admittance control scheme may be used to set the admittance levels based on short term (e.g., next day) weather predictions. Finally, the DRS[h] will correspond to an apparatus/device that performs dynamic solar radiation reduction for each hour of the day; hence, a control scheme with active weather condition tracking will be required to address the optimum solar radiation admittance levels.

In section 3, we showed that the North-facing zone did not provide any energy saving improvements when we simulated the zone under reduced solar radiation admittance scenarios (Fig. 2(a)). In fact, for the North-facing zone the reference scenario itself was the best energyperforming scenario. However, under dynamic regulation strategies (DRS) we notice energy savings even for the North-facing zone (Fig. 5(a)). More specifically, North-oriented zones can save in total $\sim 1 \mathrm{kWh} / \mathrm{m}^{2} \mathrm{y}$ (e.g., $1 \%$ compared to the reference scenario) on a monthly regulation strategy, $3 \mathrm{kWh} / \mathrm{m}^{2} \mathrm{y}$ (or $4 \%$ ) on a per day regulation strategy and $13 \mathrm{kWh} / \mathrm{m}^{2} \mathrm{y}$ (or $18 \%$ ) on a per hour regulation strategy. The savings observed on a DRS[h] are significant and to our knowledge never reported before. In addition, for the East-facing zone each regulation strategy contributes a total of $15 \mathrm{kWh} / \mathrm{m}^{2} \mathrm{y}$ (or $14 \%$ ) energy savings for the DRS[m], $20 \mathrm{kWh} / \mathrm{m}^{2} \mathrm{y}$ (or $19 \%$ ) for DRS[d], and $35 \mathrm{kWh} / \mathrm{m}^{2} \mathrm{y}$ (or 33\%) for DRS[h]. The South orientation zone provides significant energy savings as well, where the monthly regulation strategy leads to $18 \mathrm{kWh} / \mathrm{m}^{2} \mathrm{y}$ (or $21 \%$ ) total energy savings, the daily regulation strategy translates to $22 \mathrm{kWh} / \mathrm{m}^{2} \mathrm{y}$ (or $25 \%$ ) energy consumption reduction, and the hourly regulation strategy results in a $33 \mathrm{kWh} / \mathrm{m}^{2} \mathrm{y}$ (or $37 \%$ ) energy reduction. The West-oriented zone shows results similar to those of the Eastoriented zone. Compared to its reference scenario the dynamic regulation strategies reduce TPER, by $18 \mathrm{kWh} / \mathrm{m}^{2} \mathrm{y}$ (a $18 \%$ improvement) for DRS[m], $22 \mathrm{kWh} / \mathrm{m}^{2} \mathrm{y}$ (a 22\% improvement) for DRS[d], and $36 \mathrm{kWh} / \mathrm{m}^{2} \mathrm{y}$ (a $36 \%$ improvement) for DRS[h]. Hence, our analysis shows that dynamic regulation strategies offer significant savings in all orientations, including the North orientation for a typical size office room in the NYC climate. Considering the number of available office spaces in office buildings the potential for total energy savings is significant, and could lead to a great energy reduction for the entire city. 


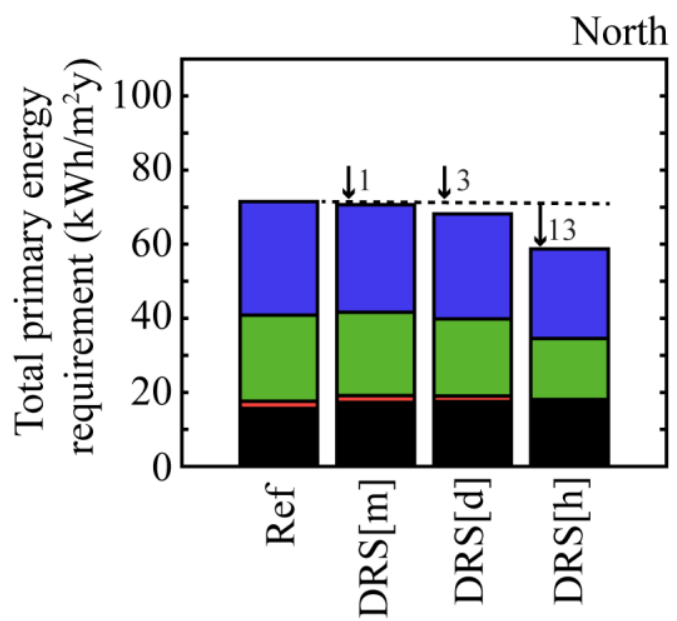

(a)

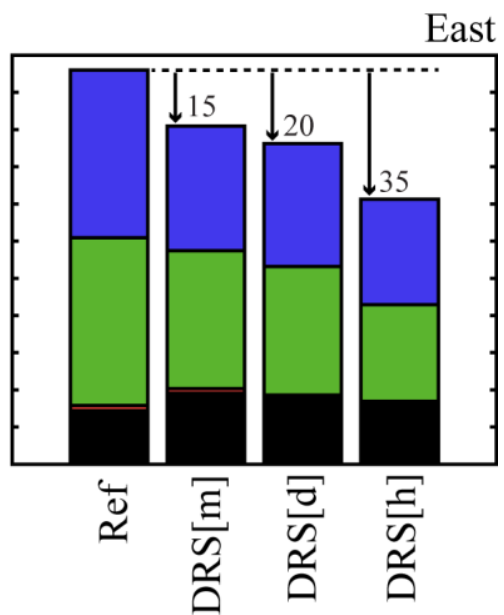

(b)

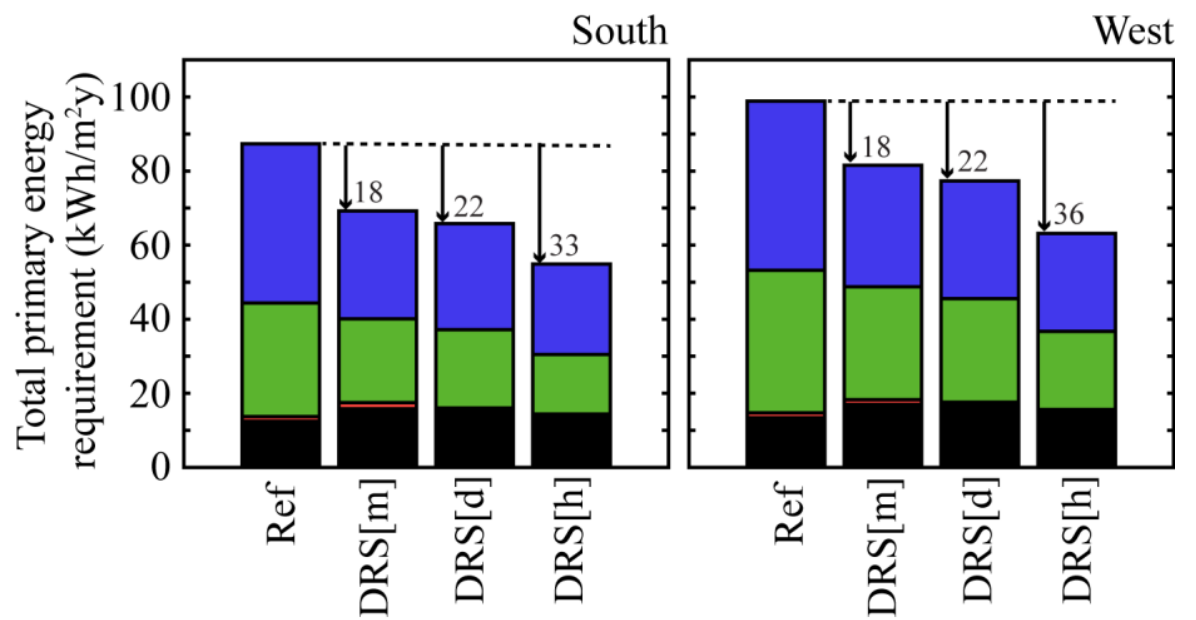

(c)

(d)

- Lights $\backsim$ Heating $₫$ Fans $₫$ Cooling

Fig. 5. Dynamic regulation strategies comparison for (a) North, (b) East, (c) South, and (d) Westfacing zones. (Note: DRS[m] is monthly, DRS[d] is daily, and DRS[h] is hourly regulation).

\subsection{Impact of potential performance limitations of dynamic fenestration technology on energy savings}

The regulation strategy results are very interesting because they show the potential of dynamic fenestrations for providing energy savings under dynamic climate conditions. As we described in the previous section, the choice of the particular strategy (e.g., DRS[m], DRS[d], DRS[h]) depends on the orientation of the zone. However, the energy savings potential from each orientation and DRS may also depend on technology performance and technology cost.

In Fig. 5, we presented results that were based on a resolution of $1 \%$ and a full dynamic range from $100 \%$ to $10 \%$ of the solar radiation admittance levels. We define as dynamic range (DR) the difference between the maximum and the minimum percentage of admitted solar radiation. Note that the maximum required solar radiation admittance should always reach the $100 \%$ for both solar radiation components. This fine resolution and large DR may be considered as an ideal performance from potential future dynamic fenestrations or devices/apparatuses that can be 
installed on existing glazings. If a future fenestration technology cannot achieve such performance metrics, it is expected that the energy savings potential will be reduced. Hence, it is very important to understand and quantify the impact on the energy savings from potential limitations on the technology performance metrics, such as reduced dynamic range and/or coarse (e.g., $5 \%$ instead of $1 \%$ ) resolution of the solar radiation admittance regulation levels.

Fig. 6 shows the direct and diffuse solar radiation admittance levels required in order to consume the least amount of energy for each month under a DRS[m] approach. We notice that the DR is smaller for the North orientation since North does not need high levels of solar radiation regulation under DRS $[\mathrm{m}]$ regulation. In addition, a balanced regulation of direct and diffuse solar radiation contributes to maximum energy savings. This is because the North-facing zone is not exposed to direct solar radiation and therefore there is no need to strongly regulate the direct component. In contrast, the other zones show that there is a dominant direct component that needs to be reduced to levels as low as $10 \%$ for maximum energy savings. The diffuse solar radiation in those cases can be kept at levels of $40 \%$ and higher.

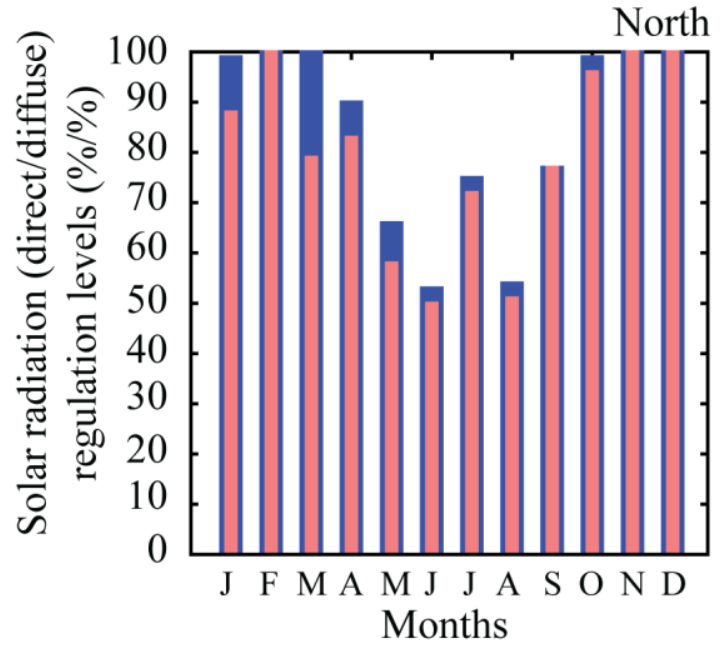

(a)

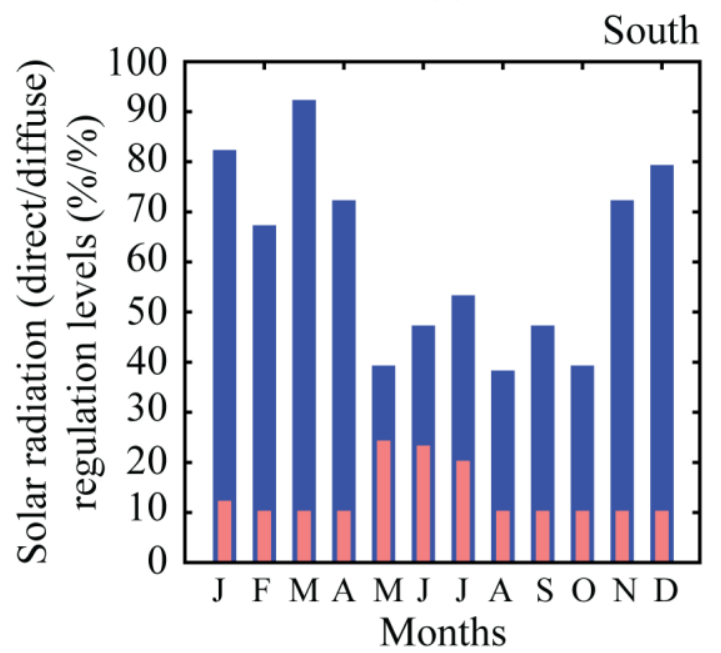

(c)

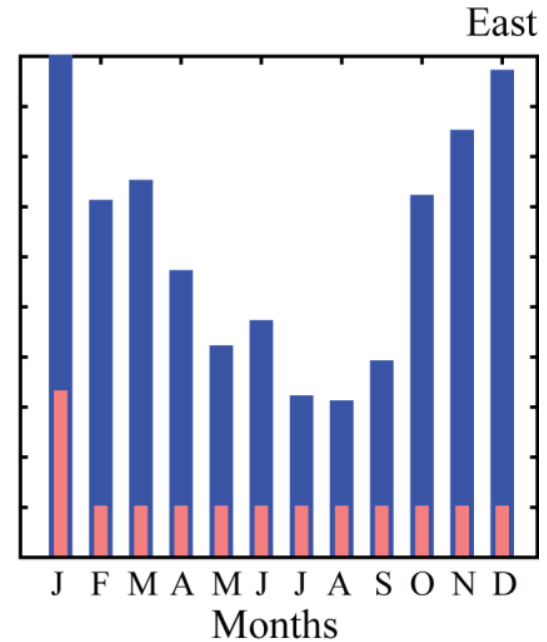

(b)

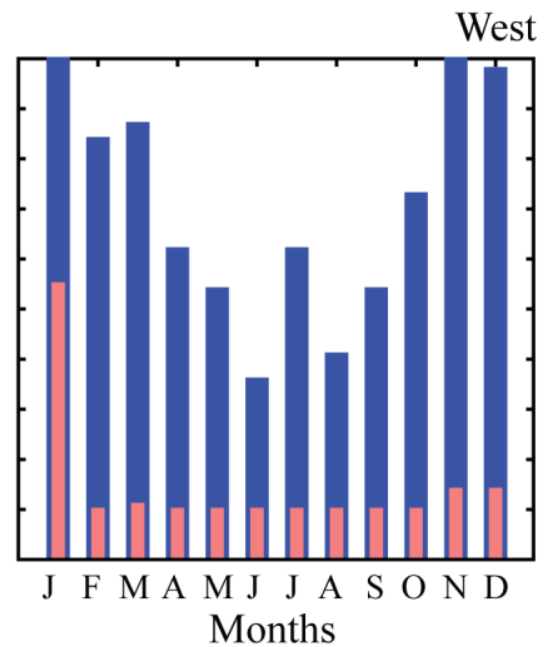

(d)

direct diffuse

Fig. 6. Dynamic range of solar radiation regulation for the DRS[m]. 
We can conclude from Fig. 6 that a wide range of solar radiation regulation is required in order to optimize the zone's energy consumption. This becomes even more complex in the case of daily (DRS[d]) and hourly (DRS[h]), where our study shows that the admittance levels can vary from a high 100/100 \% (direct/diffuse) to a low 10/10\% to achieve maximum energy savings. However, a broad DR may be technically more difficult to be achieved and fully satisfy the requirements. For this reason, we performed an additional study, where we evaluated the energy savings impact of reduced DR capabilities. We assumed that a potential future dynamic fenestration technology cannot satisfy the solar radiation reduction to a minimum of 10/10\%, as we reported in Figs. 2-6, but rather at higher levels, e.g., 20/20 \%, 30/30 \%, and up to 90/90 \%. Note that the resolution for the admitted solar radiation remains at $1 \%$. As expected, if the DR is limited, the energy savings will be reduced.

Fig. 7 shows the impact of the reduced DR, e.g., $\Delta$ (TPER) $=$ TPER $R_{\text {Reference }}-$ TPER $_{\text {DRS[h]DR }}$ on the energy savings potential when the DR cannot reach low levels of 10/10\% solar radiation regulation. We preset the results for monthly (DRS[m], Fig. 7(a)), daily (DRS[d], Fig. 7(b)), and hourly (DRS[h], Fig. 7(c)) basis. As expected, the impact of the reduced DR on the energy savings is different for the three different regulation strategies, as well as for the different zone orientations. In general, we see that this DR performance deviation does not have a significant effect on the North-facing offices or DSR[m] approaches, whereas the effect becomes more pronounced in the other orientations or for more aggressive DSR approaches (e.g., DSR[h]). It is important to note that although the reduction of DR translates to a reduction in energy savings, there is still energy saving potential. In particular, when determining whether glazing technology can regulate solar radiation down to a $40 / 40$ or $10 / 10 \%$ level, we notice that the energy saving impact is less than $5 \mathrm{kWh} / \mathrm{m}^{2} \mathrm{y}, 7 \mathrm{kWh} / \mathrm{m}^{2} \mathrm{y}$, and $10 \mathrm{kWh} / \mathrm{m}^{2} \mathrm{y}$ for the DRS[m], DRS[d], and DRS[h], respectively.

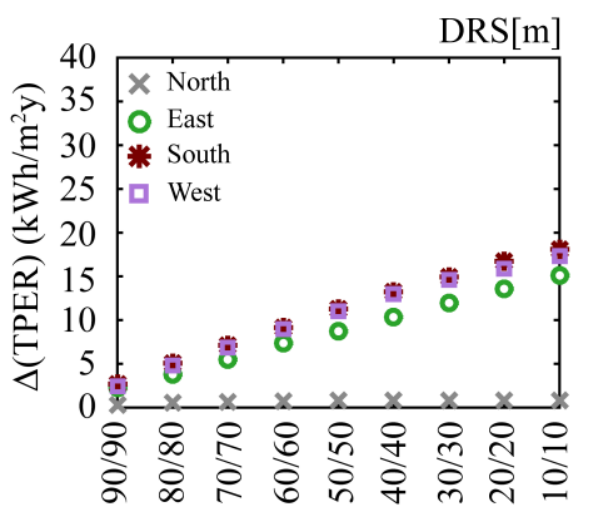

Subset scenarios

(a)

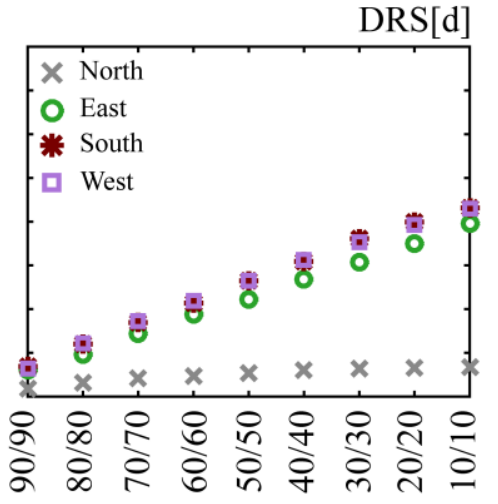

Subset scenarios

(b)

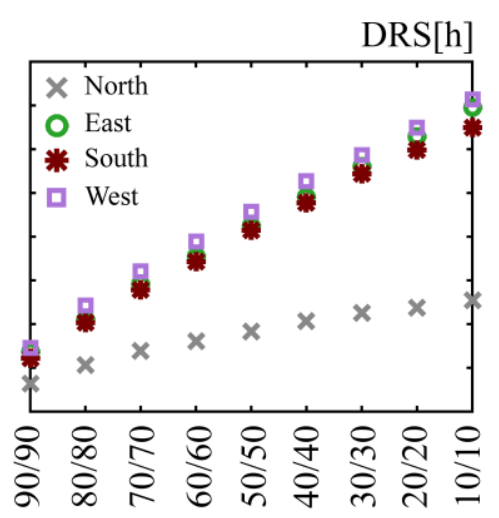

Subset scenarios

(c)

Fig. 7. Solar radiation dynamic range impact-analysis, showing the annual $\Delta$ (TPER) change if a reduced DR of the regulated solar radiation is achieved.

The above analysis and results are important for companies developing next generation fenestration solutions, as well as for engineers/architects who work at applied projects. From the companies' point of view, the analysis provides guidelines and performance targets. It can help companies make decisions about scientific and engineering investments for product development. For example, consider a glazing company that finds out that the cost and effort to 
achieve a minimum admittance level of $10 / 10 \%$ is considerably higher than that for a $20 / 20 \%$. Understanding the energy savings impact difference can help the product development team take proper action and develop a cost-effective solution. In addition, for an engineering/architecture team that is trying to identify best practices for a particular space, the above analysis can lead to important decision-making as well. For example, for a particular office space and orientation an engineer/architect may choose among the three DRS approaches as well as among different solar regulation levels. The decision will strongly depend on the potential return of investment (ROI) under a particular initial capital investment for the installation and/or operation of an active fenestration technology. As an example, consider that an engineer/architect may choose to use a potentially less expensive DRS[m] approach for a West-facing office space if that ROI is better than in the case of a DRS[h]. Note that although the absolute energy savings potential is lower with DRS[m], the initial cost and operational cost compared to a DRS[h] solution may be much lower, leading to a faster ROI for the user.

In the discussion above, we have assumed that future technology might be capable of regulating the admitted solar radiation in steps of $1 \%$. However, this may not be technically or economically feasible. Hence, we also evaluated the impact of a coarser solar regulation resolution on energy savings. Fig. 8 shows the impact on the $\Delta$ (TPER) for an admittance level resolution of $1 \%$ (same as in the results of Fig. (c)), 5\% and 10\%, for the East-facing zone and the DRS[h] case. Similar results are obtained for the other orientations and the other DRS approaches. We can conclude that the resolution step of the solar radiation admittance is not a critical parameter. We believe that this is an important conclusion as it can relieve some of the requirements of future dynamic fenestrations, since they can operate in a coarse mode (e.g., 10\% solar radiation regulation resolution) and thus presumably would be less expensive and easier to be achieved from a technology point of view.

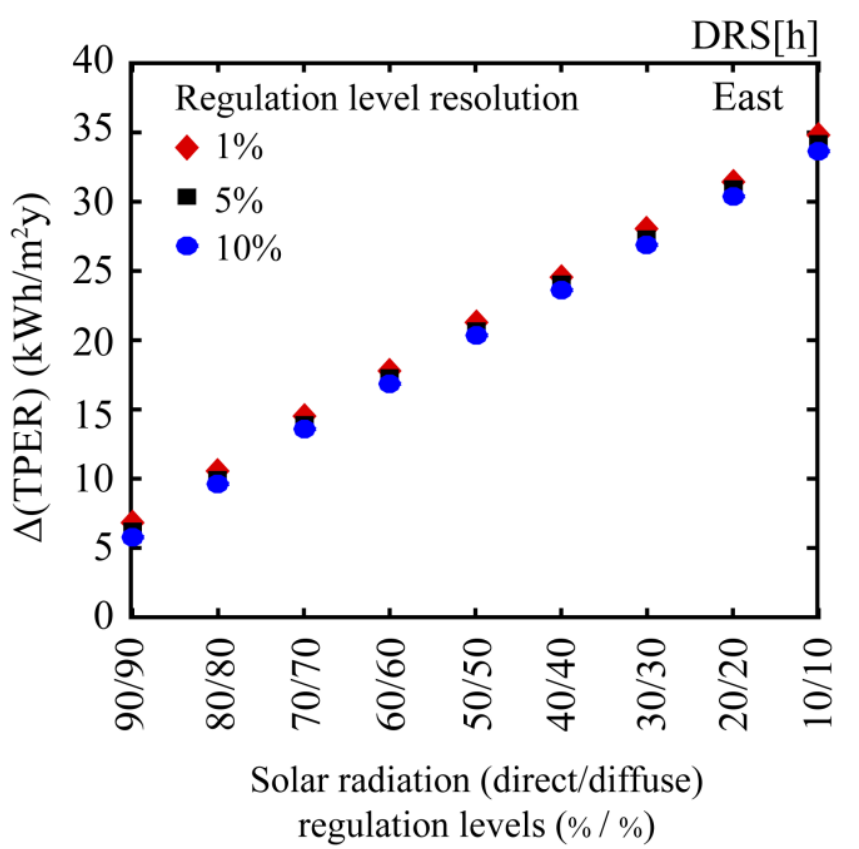

Fig. 8. Impact of solar radiation regulation resolution on TPER. Insignificant differences are shown for the different solar radiation regulation level resolution. 


\section{Conclusion}

We have presented a methodology that quantifies the impact of the individual control of the two components of solar radiation in the total energy potential. We performed the analysis for a typical office space in NYC using typical geometrical, HVAC, lighting, and occupancy schedules [12-15]. In particular, we have quantified the impact of different admittance levels of the two components of the solar radiation in the four different orientations, derived optimum admittance levels, as well as dynamic ranges and resolution of admittance levels. In addition, we have evaluated the impact on the potential total primary energy requirements under limited performance for future dynamic fenestration technology solutions. This analysis can also assist in understanding the required specifications for the development of future solutions.

In particular, our analysis shows that it is important to independently control the reduction of the two solar radiation components. The direct component affects the energy consumption more than the diffuse component. The response time of the dynamic regulation plays a significant role on energy savings in office buildings. Aggressive dynamic regulation strategies (DRS) contribute to more energy savings, e.g., DRS[h] offers from $18 \%$ to $37 \%$ savings compared to DRS[m] that offers from $1 \%$ to $18 \%$ among the different orientations. We also showed that having a full dynamic range from $100 \%$ to $10 \%$ solar radiation regulation provides maximum energy savings, since it provides maximum flexibility on the regulation strategies. Deviation of the dynamic fenestration from this ideal DR was also evaluated. We showed that performance deviation does not have a significant effect on the North-facing offices or in DSR[m] approaches, whereas the effect becomes more pronounced in the other orientations or for more aggressive DRS approaches (e.g., DRS[h]). We also evaluated the impact of a coarse admittance level (e.g., $5 \%$ or 10\%) control compared to a fine control of 1\%. Our analysis shows that the impact on energy performance is very small and we can conclude that this feature (e.g., fine control of admittance levels) is not as critical for future dynamic fenestrations. The critical parameter is the minimum solar radiation admittance level that the dynamic fenestration can achieve.

In our current work, we have included monthly, daily, and hourly solar regulation strategies. However, in our future work we will also evaluate more aggressive controls, such as five and ten minute intervals. In addition, we will transit from the standard TMY (Typical Meteorological Year) weather file to include real-time weather conditions. Lastly, future work includes the evaluation of the energy performance of a thermal zone when a portion of the direct solar radiation is transformed to diffuse solar radiation, via the use of diffusive optical properties of various elements on the window level. Furthermore, our future work will involve applying our methodology, using the climate classification of Briggs et al [16], to all major U.S. climates and their representative cities. Solar path, insolation availability, and solar irradiance vary across the U.S. Therefore, different cities are affected differently by the sun and provide more or less opportunity for energy savings. This work will assist in the development of guidelines for future dynamic fenestration technology in the different climate zones, as well as the development of dynamic devices/apparatuses that can be installed on existing glazings in order to provide energy saving opportunities. 


\section{Acknowledgement}

The authors would like to thank the New York State Energy Research and Development Authority, and the NSF sponsored IUCRC, Sustainable Integrated Buildings and Sites (SIBS) for partial support of the project. The authors would like to thank the anonymous reviewers for their valuable comments and suggestions.

\section{References:}

[1] A. Lovins, The Negawatt Revolution. http://www.rmi.org/Knowledge-Center/Library/E9020_NegawattRevolution, Rocky Mountain Institute, Boulder, CO, (1990), (accessed 10.01.2016).

[2] Building Energy Data Book, United States Department of Energy, http://buildingsdatabook.eren.doe.gov/docs\%5CDataBooks\%5C2010_BEDB.pdf, (2011), (accessed 10.01.2016).

[3] J. Carmody, S. Selkowitz, E.S. Lee, D. Arasteh, T. Willmert, Window systems for high-performance buildings, W. W. Norton \& Company Inc, New York (2004).

[4] F. Favoino, M. Overend, Q. Jin, The optimal thermo-optical properties and energy saving potential of adaptive glazing technologies, Appl. Energy (156) 2015 1-15.

[5] F. Hammad, B. Abu-Hijleh, The energy savings potential of using dynamic external louvers in an office building, Energy Build 42 (10) (2010) 1888-1895.

[6] I. Susorova, M. Tabibzadeh, A. Rahman, H.L. Clack, M. Elnimeiri, The effect of geometry factors on fenestration energy performance and energy savings in office buildings, Energy Build. 57 (2013) 6-13.

[7] A. Tzempelikos, A.K. Athienitis, P. Karava, Simulation of façade and envelope design options for a new institutional building, Sol Energy 81 (2007) 1088-1103.

[8] T. Ihara, A. Gustavsen, B.P. Jelle, Effect of façade components on energy efficiency in office buildings, Appl Energy 158 (2015) 422-432.

[9] K.J. Chua, S.K. Chou, An EETV-based approach to improving the energy performance of commercial buildings, Energy Build 42(4) (2010) 491-499.

[10] A. Vlachokostas, N. Madamopoulos, A new methodology for quantifying the impact of direct and diffuse solar radiation on energy savings in commercial office spaces, Energy Procedia 78 (2015) 2214-2219.

[11] Department of Energy, Weather data for New York, LaGuardia AP 725030(TMY3), USA, Available at: http://apps1.eere.energy.gov/buildings/energyplus/cfm/weather_data3.cfm/region=4_north_and_central_amer ica_wmo_region_4/country=1_usa/cname=USA\#NY (accessed 10.01.2016).

[12] C. Buratti, E. Moretti, E. Belloni, F. Cotana, Unsteady simulation of energy performance and thermal comfort in non-residential buildings, Build Environ. 59 (2013) 482-491.

[13] J. Mardaljevic, A. Nabil, Electrochromic glazing and facade photovoltaic panels: a strategic assessment of the potential energy benefits, Light Res. Technol. 40 (2008) 55-76.

[14] EnergyPlus ${ }^{\mathrm{TM}}$ 8.2, Input Output Reference documentation, (2015) 364.

[15] H. Shen, A. Tzempelikos, Sensitivity analysis on daylighting and energy performance of perimeter offices with automated shading, Build Environ. 59 (2012) 303-314.

[16] R.S. Briggs, R.G. Lucas, T. Taylor, Climate Classification for building Energy Codes and Standards: Part 2Zone Definitions, Maps and Comparisons, Technical and Symposium Papers, In: ASHRAE winter meeting, January 31, Chicago, IL, USA; (2003).

[17] Sustainable Energy Authority of Ireland (SEAI), http://www.seai.ie/Your_Business/Public_Sector /FAQ/Calculating_Savings Tracking_Progress/What_are the conversion factors used to calculate TPER. $\underline{\mathrm{html}}$ (accessed 10.01.2016). 